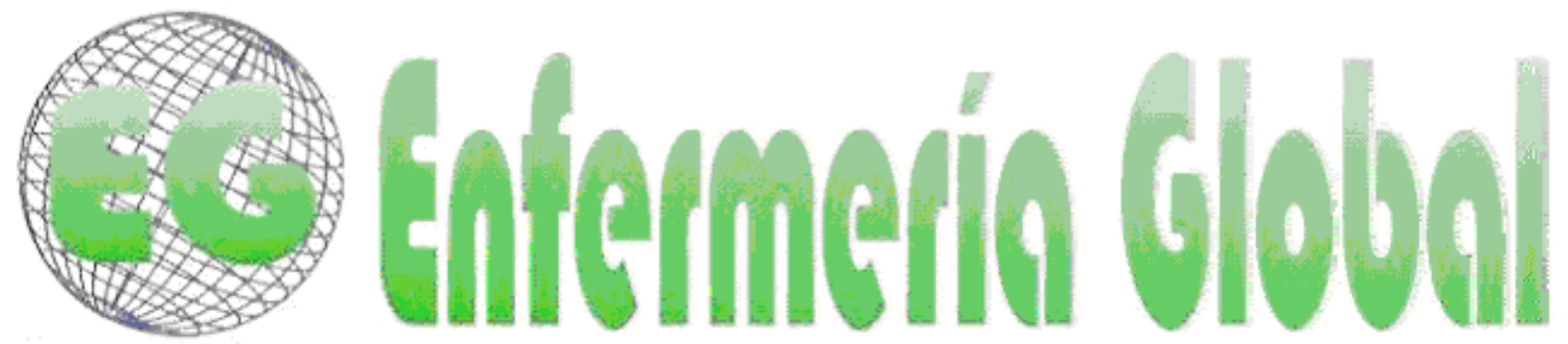

\title{
Atención a pacientes con úlcera venosa: percepción de los enfermeros de Estrategia de Salud Familiar
}

Cuidados aos portadores de úlcera venosa: percepção dos enfermeiros da Estratégia de Saúde da Família

\section{*Lopes de Figueiredo, M. ** Bonato Zuffi, F.}

\author{
*Enfermera. E-mail: marialf09@hotmail.com **Magistrer en Enfermería en Salud Pública. Profesora \\ Asistente del Curso de Graduación en Enfermería. Universidad Federal do Triângulo Mineiro - \\ Uberaba MG. Brasil.
}

Palabras clave: enfermería; ùlcera de pierna; úlcera varicosa; Salud de la Familia.

Palavras-chave: enfermagem; ùlcera de perna; úlcera varicosa; Saúde da Família.

Keywords: nursing; leg ulcer; varicose ulcer; Family Health.

\section{RESUMEN}

Se trata de un estudio descriptivo con un abordaje cualitativo, que tiene como objetivo identificar el conocimiento sobre los cuidados necesarios a personas portadoras de úlcera venosa, presentados por los enfermeros de los equipos de Estrategia de Salud de la Familia del distrito sanitario II de UberabaMG, y describe las percepciones de esos enfermeros sobre esos cuidados. Después de la aprobación en el Comité de Ética y autorización de la secretaría de salud del municipio, fueron recogidos los datos a través de entrevista semi-estructurada con 13 enfermeros. La técnica de análisis utilizada fue la propuesta por Minayo, de donde surgieron tres categorías: conocimiento adecuado, insuficiente y desconocimiento en relación a la etiología de la úlcera venosa, los cuidados y orientaciones al portador y si hay una abordaje integral. Los datos apuntan a la necesidad de una capacitación de los profesionales en relación al tema, mejores condiciones de trabajo, adopción de un protocolo de tratamiento y sustitución del modelo biomédico por una visión mas integral del cuidado.

\section{RESUMO}

Trata-se de um estudo descritivo com abordagem qualitativa, que objetivou identificar o conhecimento sobre os cuidados necessários às pessoas portadoras de úlcera venosa, apresentados pelos enfermeiros das equipes de Estratégia de Saúde da Família do distrito sanitário II de Uberaba-MG, e descrever as percepções desses enfermeiros sobre esses cuidados. Após a aprovação no Comitê de Ética e autorização da secretaria de saúde do município, foram coletados os dados através de entrevista semi-estruturada com 13 enfermeiros. A técnica de análise utilizada foi a proposta por Minayo de onde surgiram três categorias: conhecimento adequado, insuficiente e desconhecimento em relação à etiologia da úlcera venosa, os cuidados e orientações ao portador e se há uma abordagem integral. Os dados apontam a necessidade de uma capacitação dos profissionais em relação ao tema, 
melhores condições de trabalho, adoção de um protocolo de tratamento e substituição do modelo biomédico por uma visão mais integral do cuidado.

\section{ABSTRACT}

This is a descriptive study with a qualitative approach, which aimed to identify the knowledge on the care necessary for bearer people of venous ulcer, presented by nurses from the teams of the Estratégia de Saúde da Família do Distrito Sanitário 2 from Uberaba-MG, and to describe the perceptions of those nurses on the care. After the approval of the Ethics Committee and approval by the department of health from the city, data were collected through semi-structured interviews with 13 nurses. THE technique of analysis used was the one proposed by Minayo, where have appeared three categories: adequate knowledge, insufficient and illiteracy in relation to the etiology of venous ulcer, the care and guidance to the bearer, and if there is an integral approach. The data indicate the need for a training of the professionals in relation to the topic, better working conditions, the adoption of a protocol of treatment and replacement of the biomedical model by a more integral vision from the care.

\section{INTRODUCCIÓN}

La ulcera venosa (UV) tiene como principal etiología la insuficiencia venosa crónica. Cuando las válvulas de las venas de las piernas están damnificadas, el flujo sanguíneo, que debería ocurrir de las venas superficiales para las venas profundas, pasa a fluir sin dirección, ocasionando hipertensión venosa. Esta, a su vez hace que los capilares se tornen más permeables, permitiendo que macromoléculas como fibrinógeno, hematíes y plaquetas pasen por el espacio peri vascular. Este evento causa alteraciones cutáneas como edema, eczema, hiperpigmentación y lipodermatoesclerosis haciendo con que la piel quede más sensible y propicia a una lesión. ${ }^{1,2}$

La UV puede iniciarse de forma espontánea o por un trauma. Cuando se inicia de forma espontánea, generalmente se localiza arriba de los maléolo internos; cuando por traumatismo, lo que es más común, surgue en otras regiones como la fase anterior y lateral de la pierna, o en el pié. ${ }^{1,3,4,5}$

Está presente en individuos de diversas fases etarias. Aunque la susceptibilidad aumenta de forma proporcional a la edad. Se observa mayor prevalencia de 60 a 80 años siendo que $72 \%$ de las personas se vuelven portadoras de su primera úlcera a los 60 años; $22 \%$ a los 40 años y $13 \%$ anted de los 30 . 5,6,7

En un estudio brasileño en el municipio de Botucatu (SP), se observó el predominio en la fase etaria entre los 40 y 60 años (75\%) y en el municipio de Belo Horizonte (MG) la población entre 51 y 70 años representó $62,5 \%$ de los portadores. ${ }^{1,3}$

Se estima que en Brasil 3\% de la población presenta la lesión siendo que ese índice llega a $10 \%$, en el caso de asociaciones con enfermedades de base como la diabetes mellitus. ${ }^{3,8,9,10}$ Una revisión bibliográfica dió a conocer que en Europa, Australia y Estados Unidos la prevalencia varió de 0,11 a 0,18 en la población. ${ }^{2}$ Mundialmente se estima que la prevalencia de la lesión sea de 0,5 a 2\% de la población. ${ }^{10,11}$

A pesar de no presentar índices significativos de mortalidad, la morbosidad es alta debido al tiempo prolongado de cicatrización, la baja calidad de vida de los portadores durante este periodo, dolor, incapacidad parcial en la realización de algúna actividad diaria de rutina, baja autoestima, depresión y aislamiento social ${ }^{12}$ y elevado índice de recaída. Un estudio en la ciudad de Londres describe que 55\% de los pacientes 
tienen úlcera por más de una año, y 35\% por más de 18 meses siendo que, el índice de recaída fue de $60 \%{ }^{1,2,5}$

La combinación de factores como la gran incidencia en la fase de edad financieramente productiva, la larga duración del tratamiento y elevado índices de recaída, componen un agravante para el sistema público de salud desde el punto de vista económico. En Brasil la UV es la 14aㅗ causa de abandono temporal del trabajo y $32^{\mathrm{a}}$ causa de abandono definitivo. ${ }^{8}$

Se estima que en los Estados Unidos, el costo del tratamiento de la UV es de un billón de dólares por año. A pesar de que los estudios nacionales sobre incidencia y prevalencia de UV son escasos, podemos inferir que los costos para el Estado son elevados, visto que los portadores de UV son, en su mayoría, usuarios del servicio público de salud, y como ya fue dicho, el tiempo de cicatrización es largo y las recaídas frecuentes.

El estudio realizado en 2010 en un laboratorio de heridas vasculogénica en el municipio de Rio de Janeiro, se calculó un costo aproximado de curativos de úlceras limpias e infectadas, teniendo en cuanta los gastos con materiales y horas de trabajo del profesional de enfermería. Se observó que el costo medio total de curativo limpio fue de $R \$ 32,50$ y el costo medio total de curativo infectado, $R \$ 45,54^{8}$

La terapia de utilización de cobertura y curativo, juntamente con la terapia de compresión -por medio de la utilización de medias calcetines, vendas, botas Unna- y las intervenciones quirúrgicas cuando son necesarias, son las principales estrategias en el tratamiento de la UV. ${ }^{1,5}$ Una tesis doctoral realizada en Brasil resultó en una propuesta directriz para el tratamiento tópico de la úlcera venosa que abarca: evaluación del paciente y de su herida, documentación de su historial clínico, cuidados con la herida, y la piel de alrededor, indicación de la cobertura, uso o no de antibióticos, mejoría del retorno venoso y prevención de recaída, encaminamiento de los pacientes y capacitación profesional. ${ }^{1}$

La atención básica de salud (ABS), a través de la Estrategia de Salud de la Familia (ESF) tiene el papel central en relación a la úlcera venosa. Tiene como objeto de trabajo la familia y sus relaciones con el medio, la ESF trabaja en una perspectiva de [...] asistencia integral, continua, con resolución y buena calidad a las necesidades de salud de la población adscrita.[...] ${ }^{13}$

Es la ESF en la figura del enfermero y su equipo la que va a identificar a los portadores de UV, evaluarlos en todos sus aspectos - condiciones socioeconómicas, actividad laboral, factores de riesgo, factores que dificultan el tratamiento, hábitos de vida- así como definir la manera del tratamiento de la lesión, tornando así al portador y a su familia sujeto activo de ese proceso. ${ }^{13,14}$

Lo que se observa es que el usuario, cada vez que busca el servicio es atendido por un profesional diferente, la mayoría de las veces sin preparación o desactualizado, prestando un cuidado empírico, haciendo que el tratamiento no tenga una continuidad y no sea resuelto. El modelo biomédico prevalece, reduciendo el foco de cuidado a la herida sin una abordaje integral del individuo. ${ }^{1,4,15}$

La ABS que debería ser una puerta de entrada en el sistema de salud se muestra ineficaz y estos buscan otro nivel de asistencia, sobrecargando así dicho sistema. ${ }^{16} \mathrm{~A}$ 
pesar de eso, las fallas en la referencia y contra referencia crean un espacio en el tratamiento lo que acentúa las posibilidades de recaída.

Ante estos factores prevalece la necesidad de crear un protocolo que ofrezca directrices para un cuidado más específico, con una asistencia multidisciplinar y espacio para la actuación del portador y sus familiares de forma que el tratamiento tenga un resultado eficaz y con menores costos. ${ }^{1,5,10}$ Para que eso ocurra los profesionales deben estar capacitados y actualizados en todo lo que respecta a la úlcera venosa. Se buscó conocer cómo ha sido prestada esa asistencia que nos propusimos realizar en el estudio, cuyo objetivo es identificar el conocimiento sobre los cuidados necesarios a los portadores de UV, presentados por los enfermeros de los equipos de ESF del distrito sanitario II de Uberaba- MG y describir las percepciones de estos enfermeros sobre tales cuidados.

\section{MATERIALES Y MÉTODOS}

Se trata de un estudio descriptivo con un enfoque cualitativo. El proyecto de investigación fue sometido a evaluación dle Comité de Ética e Investigación con Seres Humanos de la Universidad Federal del Triángulo Minero y aprobado sobre el parecer $\mathrm{n}^{\circ} 1574$, y su realización fue realizada por la Secretaría Municipal de Salud de Uberaba.

Los datos fueron recogidos en 9 unidades del distrito sanitario II, del municipio de Uberaba, MG, que ofrece apoyo a 14 ESFs. Los sujetos del estudio fueron 13 enfermeros de los respectivos ESFs, siendo que dos de esas ESFs, las rurales, contaban con un mismo enfermero

La recolección de datos ocurrió en el periodo de enero a abril de 2011. Fue utilizado un guión de entrevista semiestructurada, con 4 preguntas abiertas e individuales: ¿Qué entiende usted por ulcera venosa (UV)? ¿Cuáles son los cuidados que considera necesario tener con la UV? ¿Cuáles son las orientaciones necesarias para la persona que tiene UV? ¿Durante los cuidados con la persona portadora de UV, usted trata otros aspectos aparte de la herida?

Las entrevistas fueron previamente programadas y ocurrieron en su mayoría en la propia unidad de salud donde la ESF estaba instalada, excepto la de los dos enfermeros de la zona rural que ocurrieron en la Biblioteca Municipal de Uberaba.

Los 13 enfermeros consintieron en participar del estudio, leyeron y firmaron un acuerdo de consentimiento en el que constaban dos objetivos de estudio, la ausencia de riesgos y cargos, los posibles beneficios, el secreto absoluto de sus identidades, ya que serían identificados por número, y la libertad de no participar e interrumpir la participación en el estudio cuando lo desearan. Todas las entrevistas fueron grabadas en medio digital con autorización de los participantes, y posteriormente fueron transcritas

Los datos fueron analizados sobre la propuesta de Minayo $^{22}$ en, la que la operacionalidad de análisis temáticos se cubría en tres etapas: Pre-análisis, Experimentación del material y Análisis de los resultados obtenidos 


\section{RESULTADOS}

Fueron entrevistadas 11 enfermeras y 2 enfermeros, con tiempo de prácticas entre 2 y 19 años -11 enfermeros con tiempo de práctica de 2 a 7 años, y otros 2 más con más de 10 años de práctica-. En cuanto al tiempo de trabajo en la ESF la variación fue de 1 a 10 años- 6 enfermeros trabajaban en la ESFs menos de 2 años, 6 enfermeros trabajaban entre 3 a 6 años y un enfermero trabajaba hace 10 años. De los entrevistados 5 trabajaban exclusivamente con ESF desde que se recibieron y los otros 8 enfermeros trabajaban en otras aéreas de enfermería como técnicos de enfermería hospitalaria y salud de la familia, enfermeros de la red, enfermeros de home care, enfermeros de guardia en Unidades de Urgencias, supervisores de prácticas y gerentes de salud.

De este análisis surgieron tres categorías: conocimiento adecuado, conocimiento insuficiente y desconocimiento del tema.

Fue considerado conocimiento adecuado cuando el entrevistado responde correctamente a las preguntas guiadas; conocimiento insuficiente cuando relata menos de cinco afirmaciones verdaderas y desconocimiento cuando respondía de manera inadecuada o desconocía totalmente el tema de la preguntas.

En la primera pregunta guiada-

¿Qué entiende usted por ulcera venosa?- 5 enfermeros dieron respuestas adecuadas, 7 enfermeros presentaron conocimiento insuficiente sobre el tema y uno demostró desconocimiento total.

Conocimiento adecuado:

La úlcera venosa es una herida, una lesión causada por alteraciones, deficiencia, en el drenaje venoso (E09)

(...) comienza con una estasis venosa(...) Comienza generalmente con una herida pequeña, la piel generalmente es una piel que la persona rasca mucho, ella está muy irritada, más seca, generalmente la región pica y allí al rascar o pegar abre una lesión. (E13)

(...) acompañamiento humanizado, usted no va a mirar solo la herida del paciente, usted va a ver donde ella vive, la parte social, si tiene alguien para ayudarla en su casa, tiene que ver si ella tiene condiciones de comprar los medicamentos que debe tomar. Toda esa parte social. (E12)

Conocimiento insuficiente:

Sí, claro, porque existen varios aspectos, ¿no? para que una herida cicatrice. No es solo la herida, no vamos evaluar la herida, vamos a evaluar al paciente y cuando usted evalúa al paciente, usted tiene que evaluar los aspectos nutricionales, los aspectos de la enfermedad de base y también la causa de esa ulcera (...) (E10)

Bueno, normalmente ya es una persona que tiene una mala circulación, se puede aprovechar la oportunidad para hacer otras investigaciones, como hipertensión, diabetes, y otro tipo de orientación, ¿no? (E04) 
Desconocimiento:

Es... entonces, hidratación, cambio a posición de decúbito (...)

Observamos a lo largo de las entrevistas la repetición de algunos datos importantes en relación a las dificultades enfrentadas por los enfermeros. En ellas 8 enfermeros manifestaron falta de estructura, material, estandarización del tratamiento y capacitación profesional para ofrecer una buena asistencia a los portadores de UV:

(...) hay mucho paciente principalmente en el PSF que atendemos con ese problema, ¿no? (...) Yo por ejemplo, conozco algunos profesionales que no tiene tanta facilidad para tratar la úlcera venosa, y mucho menos hacer curativos, orientación del curativo, conozco pacientes que a veces llegan de otras áreas que nunca recibieron una orientación, ¿no? ¿Cómo hacer un curativo?, ¿Cómo mantener el miembro en posición elevada o no?... Entonces así, a veces, falta, vamos a suponer, una capacitación mayor (...) (E03)

2 enfermeros hablaron al respecto de la cultura popular que envuelve el tratamiento de la UV:

¿Sabes por qué? Ellos tienen una creencia muy grande de 'ah me voy a bañar con esto, me voy a bañar con aquello', con hierbas de no sé que, ¿entiendes? (E06)

7 enfermeros citaron en algún momento de la entrevista qué capacitan los cuidadores y también al propio portador de UV.

(...) entonces lo hacemos en sus domicilios, hacemos el curativo con el paciente, la familia, orienta, ¿no? Para poder y... que sea mejor ejecutado, y para poder acompañar hasta que mejore, ¿no? (E01)

(...) El paciente tiene que tener responsabilidad también, ¿no? No es solo responsabilidad del profesional de la salud, ¿no? Entonces tiene que haber más personas con el paciente ¿no? Por el paciente (...) la familia también, nosotros orientamos a los familiares que van a realizar el curativo(...) (E08)

\section{DISCUSIÓN}

La mayoría de los enfermeros entrevistados (8) demostraron poco conocimiento sobre la etiología de la UV, confundida a veces como úlcera de otras etiologías como la de las arterias y la úlcera por presión. También aquellos que respondieron correctamente no mencionaron sobre el proceso etiológico, citando apenas el factor central de la insuficiencia venosa. El conocimiento de la causa de la lesión es de fundamental importancia en la elección del tratamiento. Sin esa diferenciación el cuidado se transforma general, lo que tarda o impide la cicatrización. 4,14,15,17

Los cuidados con el portador de la UV incluyen la evaluación del paciente y su herida, realizando un seguimiento, identificando factores de riesgo relacionados con los hábitos de vida, herencia, actividad laboral, y el examen físico identifican la etiología de la lesión, localización, tamaño, características y factores relacionados, como dolor y edema Esa primera evolución es imprescindible, evaluación de evolución del tratamiento empleado. A pesar del examen físico, diagnostico clínico, examen de 
laboratorio también auxilian en una evaluación más completa de las complicaciones presentes en el individuo y que pueden interferir en el proceso de cicatrización. Seguidamente la terapia tópica que envuelve la limpieza y una protección más accesible al paciente y eficiente en el tratamiento y absorción, exudación, creando un ambiente favorable para la cicatrización. Esa etapa también incluye una evaluación de la necesidad de usar antibióticos sistémicos o tópicos, no estando estos últimos indicados por la literatura. ${ }^{1,18}$

La mayoría de los enfermeros (10) respondieron adecuadamente a la segunda pregunta. Las orientaciones más citadas fueron las relacionadas con los curativos y el reposo con la posición elevada de los miembros. Es interesante observar que los enfermeros que citaron el reposo, la mayoría entiende que este tiene que ser "absoluto". Caminatas leves y regulares, ejercicios específicos que mejoran el músculo de la pantorrilla, son practicas importantes en el proceso de cicatrización. $1,5,16$

Tan importante como la cicatrización es la prevención de recaídas, con el uso de la terapia compresiva y de técnicas que mejoren el retorno venoso como reposo y la posición elevada de las piernas -de dos a cuatro horas por día, de 10 a $15 \mathrm{~cm}$ ejercicios físicos específicos y la intervención quirúrgica cuando sea necesario. Solo se sencontró un registro de orientación de terapia compresiva. El enfoque multiprofesional y la incorporación del paciente y su familia en el cuidado, también son esenciales en el proceso del tratamiento. ${ }^{1,4,14,15,16}$

Todos los entrevistados respondieron en un primer momento que tratan a los pacientes en otros aspectos aparte de la herida, en tanto que, cuando ejemplifican percibimos que la mayoría cita factores relacionados con la úlcera y a su cicatrización, evidenciando así la prevalencia de una visión biomédica en el cuidado al paciente de UV. Cinco enfermeros utilizaron la expresión "ver a la persona como un todo", demostrando que entienden que es preciso prestar cuidado integral en tanto que no comprenden la dimensión de lo que es la integridad.

La UV es una lesión que compromete varias dimensiones en la vida del portador, que tiene que convivir con el dolor crónico, prejuicio en su imagen corporal, pérdida de su autoestima, depresión, aislamiento social, perjuicio en la actividad sexual, pérdida parcial de autonomía de realización de tareas domésticas, abandono de las actividades laborales o jubilación precoz. Un estudio de revisión bibliográfica reveló un análisis de por lo menos 9 artículos, la reducción de la calidad de vida en los portadores de UV, siendo el dolor lo más perjudicial.

El enfermero también desempeña una función importante al ayudar al portador de UV a reorganizar estos aspectos de su vida, y ofrecer posibilidades de adaptación y desarrollar técnicas que se adhieran al régimen terapéutico, disminuyendo el tiempo de tratamiento y haciendo que el paciente retorne a su rutina lo más rápido posible.

La integridad como directriz del SUS ( Sistema Único de Salud) debe ser una práctica inherente al ABS, así como una conducta de todos los profesionales que la componen. Eso significa ampliar el concepto de salud y cuidado colocando al usuario en todas sus dimensiones y necesidades, como enfoque del trabajo en salud. ${ }^{19}$

La estructura para dar una buena asistencia incluye un local adecuado, material, capacitación y número suficiente de profesionales, pues la desorganización 
compromete el cuidado del paciente contribuyendo a la gravedad del problema, que termina sobrecargando los demás niveles de atención y pudiendo llevar incluso al ingreso. $^{3,5,10,16}$

Al contrario de lo que se imagina, la búsqueda por tratamiento de medicina popular no tiene relación exclusivamente con la falta de recursos económicos. Lo que ocurre es que el enfoque de esas terapias es más integral, cuidando de la enfermedad del individuo en todas las dimensiones, como psicológica y espiritual. ${ }^{20}$ Este tipo de prácticas no deberán ni deben dejar de existir. Cabe a los profesionales de la salud no ignorarlas ni desmerecerlas, sabiendo conciliar el tratamiento convencional con las creencias del paciente.

El enfermero en su esencia también es un educador. En la ABS esta función es todavía más evidente, aunque es un nivel de complejidad en el cual se enfatiza la prevención, lo que significa que el sujeto también participe de su proceso de saludenfermedad y sea capaz de cuidarse a sí mismo. Responsabilizar también al paciente por mejorar en su salud, adherirse al tratamiento, mejorar su calidad de vida y evitar recaídas ${ }^{18,21}$

\section{CONCLUSIÓN}

Actualmente las UV se tornan un problema de salud pública por su creciente incidencia, la demanda de un tratamiento largo, complejo, caro, con altos índice de recaída. Exige especialización técnica científica, prestada por profesionales cualificados.

El impacto biopsicosocial en el paciente exige una visión integral y un cuidado diferenciado por parte del equipo de salud, adecuado a cada paciente. Esto repercute en una calidad de vida mejor, en la aplicación del tratamiento, en menor tiempo de cicatrización y en la reducción de gastos públicos.

En el estudio observamos que la mayoría de los enfermeros tienen bajo conocimiento con respecto a la etiología de la UV, inclusive la confunden con otros tipos de lesiones. De esa forma aplican un tratamiento general, prolongando el tiempo de cicatrización y aumentando las posibilidades de recaída. Lo que se justifica por la falta de actualización de los profesionales y también la inexistencia del protocolo de atención a las personas que presentan heridas.

A pesar que la mayoría de los entrevistados respondió correctamente a las cuestiones relacionadas con los cuidados y orientaciones, observamos que hablaron poco sobre el tema, solamente citaron acciones generales empleadas en el tratamiento de la mayoría de las lesiones. Los históricos relacionados con la prevención de recaída fueron escasos lo que demuestra la falta de preparación de los profesionales.

En relación a la integridad, la opinión de los entevistados apunta que ellos son conscientes de la importancia de "considerar al indivíduo como un todo", pero los ejemplos citados muestran que en la práctica el modelo biomédico prevalece, este está presente en todos los niveles de atención y puede estar relacionado principalmente con la formación profesional. 
A pesar del conocimiento técnico- científico es necesario que los profesionales tengan buenas condiciones de trabajo para dar una atención satisfactoria. Identificamos en más de $50 \%$ de las entrevistas, relatos de dificultades en relación a la falta de material y un local adecuado para la realización del curativo, número insuficiente de profesionales y falta de estandarización del tratamiento. Los propios entrevistados también identificaron la necesidad de capacitación y actualización más frecuente, lo que puede ser posible a través de convenios entre universidades y servicio de salud, sea por medio de residencia proyecto de extensión o investigación.

\section{REFERENCIAS BIBLIOGRÁFICAS}

1. Borges EL. Tratamento Tópico de Úlcera Venosa: proposta de uma diretriz baseada em evidências. p.36,37,38,51 .2005, 306 f. Tese de doutorado apresentada à Escola de Enfermagem de Ribeirão Preto da Universidade de São Paulo EERP/USP. Ribeirão Preto-SP.

2. Dealey, C. Cuidando de Feridas: um guia para as enfermeiras. Tratamento de Pacientes com Feridas Crônicas. 2008. 3ed. São Paulo: Atheneu; 145-46.

3. Martins D.A, Souza A.M. O perfil dos clientes portadores de úlcera varicosa cadastrados em programas de saúde pública. Cogitare Enferm 2007 Jul/Set; 12(3):353-7.

4. Abbade LPF, Lastória $S$. Abordagem de pacientes com úlcera de perna de etiologia venosa. An Bras Dermatol. 2006;81(6):509-22.

5. Angélico, R.C.P. Úlcera venosa crônica: Qualidade da assistência e o conhecimento sobre o direito a saúde. Natal; 2010. [Trabalho de Conclusão de Curso - Programa de Pós-graduação em Enfermagem - Universidade Federal do Rio Grande do Norte].

6. França L.H.G., Tavares V. Insuficiência venosa crônica. Uma atualização. J Vasc Br 2003;2(4):318-28.

7. Maffei, F.H.A. Insuficiência Venosa Crônica: Conceito, prevalência, etiopatogenia e fisiologia. In: Maffei, F.H.A.; Lastória, S.; Yoshida, W.B.; Rollo, H. Doenças vasculares periféricas. 3 ed. Rio de Janeiro: Medsi, 2002. Cap 114.

8. Mata VE, Porto F, Firmino F. Tempo e custo do procedimento: curativo em úlcera vasculogênica. R. pesq.: cuid. fundam. online 2010. out/dez. 2(Ed. Supl.):94-97.

9. Aguiar E.T., et. al. Úlcera de Insuficiência Venosa Crônica. J Vasc Br 2005;4(Supl.2):S195-200.

10. Carneiro C.M., Sousa F.B., Gama F.N. Tratamento de feridas: assistência de enfermagem nas unidades de atenção primária à saúde. Revista Enfermagem Integrada - Ipatinga: Unileste-MG - V.3 - N.2 - Nov./Dez. 2010.

11. Cunha N.A. Sistematização da Assistência de Enfermagem no tratamento de feridas crônicas. 2006, Olinda. www.abenpe.com.br acessado em 10/10/2011.

12. Macedo E.A.B., et. al. Caracterização sócio-demográfica dos pacientes com úlcera venosa atendidos em um hospital universitário. Rev enferm UFPE on line. 2010 nov./dez.;4(spe):1863-867.

13. Brasil, Ministério da Saúde: Saúde da Família: Uma Estratégia para Reorientação do Modelo Assistencial. Brasília, p. 10.1998.

14. Nunes JP. Avaliação da Assistência à Saúde dos Portadores de Úlceras Venosas Atendidos no Programa Saúde da Família do Município de Natal/RN. 2006, 131 f. Tese de mestrado apresentado ao Programa de Pós-Graduação em Enfermagem da Universidade Federal do Rio Grande do Norte. Natal, RN.

15. Deodato O.O.N. Avaliação da qualidade da assistência aos portadores de úlceras venosas atendidos no ambulatório de um hospital universitário em Natal/RN. 2007, 
104f. Tese de mestrado apresentado na Universidade Federal do Rio Grande do Norte. Natal-RN.

16.Zuffi FB. A atenção dispensada aos usuários com úlcera venosa: percepção dos usuários cadastrados nas equipes de saúde da família. P.21, 27, 35, 129 f. Tese de mestrado apresentado à Escola de Enfermagem de Ribeirão Preto da Universidade de São Paulo EERP/USP. Ribeirão Preto-SP.

17. Bersusa A.A.S, Lages J.S. Integridade da pele prejudicada: identificando e diferenciando uma úlcera arterial e uma venosa. Rev Ciência, Cuidado e Saúde, v. 3, n. 1, p. 81-92, jan./abr. 2004, Maringá.

18. Carmo S.S., CASTRO C.D., Rios V.S., Sarquis M.G.A. Atualidades na assistência de enfermagem a portadores de úlcera venosa.Revista Eletrônica de Enfermagem [serial on line] 2007 Mai-Ago; 9(2): 506-517.

19. Fontoura, R.T. Uma breve reflexão sobre integralidade. Revista Brasileira de Enfermagem 2006; 4(59):532-537.

20.Kreutz, I., Merighi, M.A. B., Gualda, D.M.R. Cuidado Popular com Feridas: Representações e Práticas na Comunidade de São Gonçalo, Mato Grosso, Brasil. Revista Ciencia y Enfermeria 2003; 1(9): 39 - 45.

21. Silva JLA, Lopes MJM. Educação em saúde a portadores de úlcera varicosa através de atividades de grupo. Revista Gaúcha de Enfermagem 2006 jun; 27 (2): 240-250

22. Minayo MCS. O desafio do conhecimento: pesquisa qualitativa em saúde. 2004. 8.ed. São Paulo: Hucitec, 269p.

23. Silva LD, Pazos AL. A Influência da dor na qualidade de vida do paciente com lesão crónica de pele. Revista de Enfermagem da UERJ. 2005; 13: 275-281.

ISSN 1695-6141

(C) COPYRIGHT Servicio de Publicaciones - Universidad de Murcia 